\title{
Settling Statistics of Hard Sphere Particles
}

\author{
X. Lei, B. J. Ackerson, and P. Tong \\ Department of Physics, Oklahoma State University, Stillwater, Oklahoma 74078-3072
}

(Received 21 August 2000)

\begin{abstract}
Direct imaging of settling, non-Brownian, hard sphere, particles allows measurement of particle occupancy statistics as a function of time and sampling volume dimension. Initially random relative particle number fluctuations, $\left\langle N^{2}-\langle N\rangle^{2}\right\rangle /\langle N\rangle=1$, become suppressed, anisotropic, and $\langle N\rangle$ dependent. Fitting to a simple Gaussian pair correlation model suggests a minute long ranged correlation leads to strong if not complete suppression of number fluctuations. Calflisch and Luke predict a divergence in velocity fluctuations with increasing sample volume size based on random (Poisson) statistics. Our results suggest this is not a valid assumption for settling particles.
\end{abstract}

DOI: $10.1103 /$ PhysRevLett.86.3300

A collection of uniformly sized hard spheres settling in a Newtonian fluid is a simple but vexing nonequilibrium problem in statistical physics. Because of the long ranged hydrodynamic interactions, the sedimentation rate decreases from the single particle or Stokes value $U_{0}$ with increasing particle volume fraction [1]. The leading order correction first calculated by Batchelor [2-4] explicitly assumes [5]: (i) a low particle Reynolds number or the neglect of inertia, (ii) consideration of only two body hydrodynamic interactions, (iii) a random particle distribution in space, and (iv) the system size infinite in the direction transverse to the settling. This result describes, with reasonable accuracy, settling in monodisperse hard sphere suspensions dominated by Brownian motion [1]. However, Calflisch and Luke found a divergence with system size for the particle velocity fluctuations calculated within the same assumptions [6]. Segrè et al. [7] provide intuition with a simple scaling argument similar in spirit to Hinch [8]. The average number of particles in a region of suspension of linear dimension $l$ is given by $\langle N\rangle=l^{3} \phi / v_{p}$, where $\phi$ is the particle volume fraction and $v_{p}=\frac{4}{3} \pi a^{3}$ is the particle volume. Random Poisson statistics implies relative number fluctuation $\sigma_{\text {random }}^{2} /\langle N\rangle=\left\langle N^{2}-\langle N\rangle^{2}\right\rangle /\langle N\rangle=1$ and a corresponding fluctuation of $\sqrt{\langle N\rangle} \Delta \rho g v_{p}$ in the mass of the region, where $\Delta \rho$ is the difference between particle and fluid densities and $g$ is the acceleration of gravity. This must be balanced by Stokes drag $6 \pi \eta l \Delta U$ implying $\Delta U / U_{0}=$ $\sqrt{\langle N\rangle} a / l=\sqrt{3 \phi l / 4 \pi a}$, where $\Delta U$ is the typical velocity fluctuation of settling particles. Naively, if $\sigma^{2} /\langle N\rangle=$ $\langle N\rangle^{-1 / \alpha}$ for large $\langle N\rangle$, then for $\alpha \geq 3$ the velocity fluctuations do not diverge with sample size. But the particle number occupancy statistics are radically modified.

The predicted velocity fluctuation divergence received both theoretical and experimental scrutiny without consensus [5,9-16]. Computer simulations support the conclusion, finding an increase in the amplitude of velocity fluctuations with system size $[9,10]$. These simulations relax assumptions (ii) and (iii), since they approximate many particle hydrodynamics and the system may evolve via hydrodynamic interactions away from initially randomized
PACS numbers: 45.70.Mg, 05.40.-a, 83.10.Pp, 83.80.Hj

configurations. On the other hand, experiments find no dependence on system size, either experiments measuring the diffusion of a test particle [11-13] or direct measurements of the velocity fluctuations [7]. Koch and Shaqfeh [14] gave the first explanation for the absence of divergent velocity fluctuations in terms of a violation of assumption (iii), where every particle has a net deficit of one particle surrounding it within a correlation range $\xi$. This condition drives the number fluctuations to zero beyond the correlation length, but the predicted correlation length scaling as $\xi \sim a / \phi$ does not agree with experiments. Theories based on a coarse grained two fluid description of the suspension $[15,16]$ produce the experimentally observed correlation length dependencies but may [16] or may not [15] address assumption (iii) directly through calculation of the static structure factor $S(k)$, which for systems in thermodynamic equilibrium relate directly to number fluctuations [17]. Finally, Brenner [5] considers the effect of walls, thus relaxing assumption (iv) while maintaining assumption (iii), to produce the experimentally observed correlation length dependence. He argues that the experiments thus far are dominated by wall effects and that cell size effects become apparent in larger sample cells. Eventually, however, increased velocity fluctuations increase particle mixing and suppress number fluctuations, quenching the velocity fluctuations themselves.

Direct experimental tests of assumption (iii) have not been reported, either by measurement of number fluctuations in a test volume, by measuring the pair correlation function $g(r)$, or by scattering experiments to measure the static structure factor $S(k)$. Furthermore, the calculations of Koch and Shaqfeh [14] indicate that the long range behavior of $g(r)$ is inversely proportional to distance, such that the dominant contribution to number fluctuations may be difficult to observe directly in $g(r)$, yet can produce profound effects on number fluctuations on large length scales. Given these difficulties we attempt and report the results of experiments measuring particle number fluctuations directly as a function of the measurement test volume size.

A vertical rectangular cell having dimension $1 \times 1 \times$ $4 \mathrm{~cm}^{3}$ contains the samples and is placed in a large stirred 
water bath controlled at $23 \pm 0.1^{\circ} \mathrm{C}$ to eliminate convection produced by temperature gradients. Without such stirring the suspension-supernatant interface becomes unsettled. A bright, incoherent, $0.35 \mathrm{~mm}$ thick sheet of light illuminates a vertical cross section of the sample near cell center. A normal to the sheet is also normal to one of the faces of the sample container and parallel to the line of sight of the imaging system. Typically a $4.5 \mathrm{~mm} \times 3.4 \mathrm{~mm}$ patch of this cross section is imaged through a Leica MZ8 telemicroscope onto a chargecoupled device (CCD), where images are captured and stored for further processing.

Figure 1 shows particle configurations for samples containing monodisperse polystyrene spheres having diameter $d=41.88 \pm 0.82 \mu \mathrm{m}$ (Bangs Labs, Inc.) and suspended with $\phi=0.004$ in polyalkylene glycol (PAG). PAG is an organic Newtonian fluid with density $\rho=0.95 \mathrm{~g} / \mathrm{cm}^{3}$ and viscosity $\eta=0.092 \mathrm{PaS}$ at $23{ }^{\circ} \mathrm{C}$. Thus the sample cell viscous damping time is order $1 \mathrm{sec}$. The Stokes velocity is $U_{0}=\frac{2}{9} a^{2} \Delta \rho / \eta \cong 0.85 \mu \mathrm{m} / \mathrm{s}$, giving total settling time of order $10 \mathrm{~h}$ and a horizontal mixing time based on estimates of $\Delta U$ from 2.5 to $5 \mathrm{~h}$. The Peclet number given by $\mathrm{Pe}=U_{0} a / D_{0}>10^{6}$, where $D_{0}$ is the Stokes Einstein diffusion constant [1], implies negligible Brownian motion. The Reynolds numbers based on particle radius $\operatorname{Re}=2 a U_{0} \rho / \eta \approx 10^{-7}$ and on maximum cell dimension $\operatorname{Re}=2 L U_{0} \rho / \eta \approx 10^{-4}$ imply negligible inertial effects. Nevertheless, immediately after mixing and extending for several hours, this slow settling process exhibits a turbulent looking velocity field as seen in Fig. 1(b). Figure 1(a) shows a single image from this early stage of settling. Figure 1(d) shows another time lapse image taken
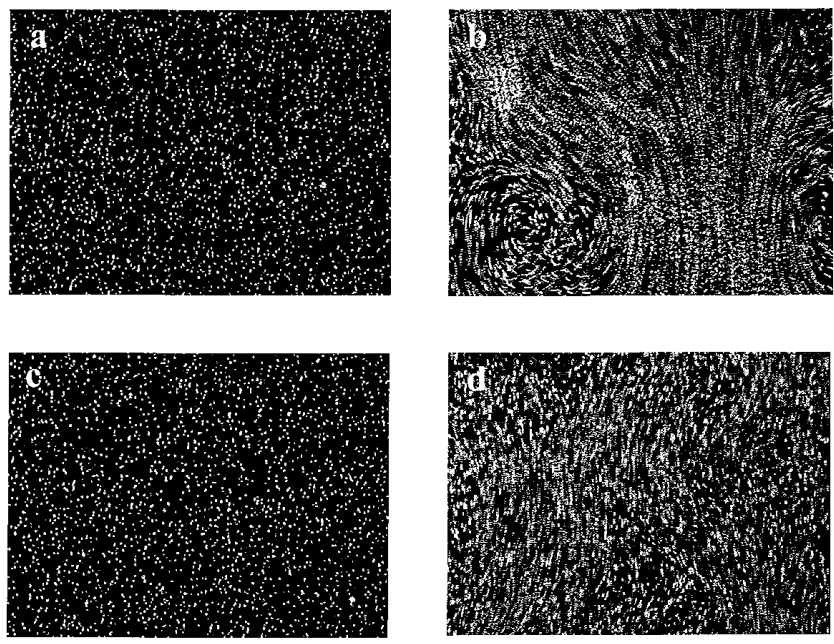

FIG. 1. Images taken during particle sedimentation: (a) A particle configuration taken $35 \mathrm{~min}$ after mixing, (b) a superposition of five sequential images taken at $30 \mathrm{sec}$ intervals beginning 30 min after mixing, (c) a particle configuration taken $5 \mathrm{~h}$ after mixing, and (d) a superposition of five sequential images taken at $30 \mathrm{sec}$ intervals beginning $5 \mathrm{~h}$ after mixing. The display area is approximately $4.5 \mathrm{~mm} \times 3.4 \mathrm{~mm}$. during the later stages of settling, and Fig. 1(c) shows a single image from this sequence. While the single images 1(a) and 1(c) show similar looking random configurations of particles, the corresponding velocity fluctuations in 1(d) are less violent than those in 1(b). Evidently there are subtle configuration differences between the two times.

In order to study these particle configurations quantitatively, we determine the particle occupancy distribution by counting the number of particles within a circle of fixed area. A distribution results from randomly positioning the circle 100 to 200 times in each of 50 independent images, obtained by viewing different regions of the light sheet in the lower third of the sample and by changing the depth of the light sheet within the sample. The time duration spanned by the 50 images is $2 \mathrm{~min}$. Measurements of the particle concentration remained constant within experimental error (number fluctuations) in both space and time. For experimental illumination reasons, we limited our viewing volume to the central third of the cell, away from the walls. Therefore the particles in the observed region should be interacting with "strong" hydrodynamic interactions according to Brenner's reasoning [5] and not be influenced by wall effects.

Figure 2 shows the occupancy distributions generated for two different sampling areas, corresponding to $\langle N\rangle=$ 6 and 58. The solid lines show the Poisson distribution for these average occupancy values. This distribution describes the earliest measurements after mixing but not at the later times, when the experimental distribution narrows relative to the Poisson distribution. Poisson statistics properly apply to point particles and ignore any particle interaction effects, such as the excluded volume for hard spheres. Particle interactions enhance or suppress number fluctuations for systems in thermodynamic equilibrium [17] according to $\sigma_{\text {thermo }}^{2} /\langle N\rangle=S(0)$, the right hand side being a constant independent of $\langle N\rangle$ provided the sampling volume exceeds the correlation range of interactions. For hard spheres with $\phi=0.004$, solution of the Percus-Yevich equation [18] gives $\sigma_{\text {thermo }}^{2}=0.97 \sigma_{\text {random }}^{2}$, indicating negligible excluded volume effects when comparing the early time distribution with the Poisson distribution. Evidently, the hard sphere volume fraction must be increased by more than an order of magnitude to produce a suppression of number fluctuations equivalent to that observed in the late stages of settling. But such comparisons implicitly assume $\sigma^{2} /\langle N\rangle$ is constant and contrary to the $\sigma^{2} /\langle N\rangle$ data shown in Fig. 3. While the early time data correspond with $\sigma_{\text {random }}\left(\sim \sigma_{\text {thermo }}\right)$, the later time data show a strong suppression of number fluctuations with increasing test volume dimension. Evidently, from Fig. 3, the relaxation time for the decay of the initial random fluctuations is a large fraction of the total settling time and is of the order of the previously estimated horizontal mixing time. This time corresponds with our observations and serves as a caution for those taking density or velocity fluctuation measurements assumed to be at steady state. 

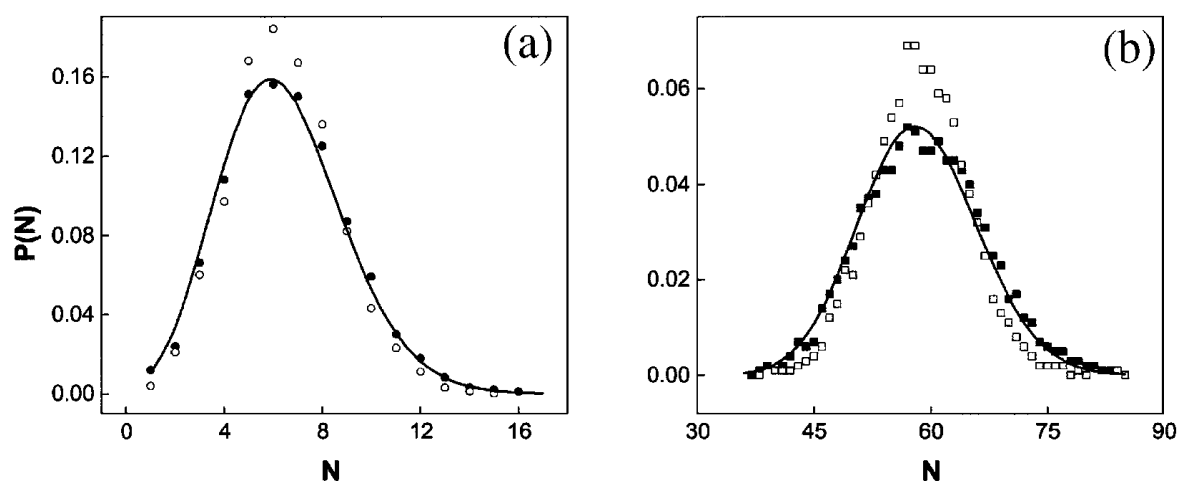

FIG. 2. Particle occupancy distributions: (a) for $\langle N\rangle=6,2$ min after mixing (filled circles) and 8 h after mixing (open circles); and (b) for $\langle N\rangle=58,2$ min after mixing (filled squares) and $8 \mathrm{~h}$ after mixing (open squares). The solid curves represent the Poisson distribution, $P(N)=\langle N\rangle^{N} e^{-\langle N\rangle} / N$ ! for the corresponding $\langle N\rangle$.

For samples having uniform density and pair correlation with translational invariance, the (nonequilibrium) number occupancy fluctuations in a finite test volume are related to the pair correlation function or structure factor as [19]

$$
\begin{aligned}
\frac{\sigma^{2}}{\langle N\rangle} & =1+\frac{\phi}{v_{p}} \int H(\bar{r})[g(\bar{r})-1] d \bar{r} \\
& =1+\frac{1}{(2 \pi)^{3}} \int[\theta(-\bar{q})]^{2}[S(\bar{q})-1] d \bar{q},
\end{aligned}
$$

where $\theta(\bar{r})$ equals unity inside the test volume and zero otherwise, $\theta(-\bar{q})$ is the Fourier transform, and $H(\bar{r})=$ $\int \theta\left(\bar{r}^{\prime}\right) \theta\left(\bar{r}-\bar{r}^{\prime}\right) d^{3} \bar{r}^{\prime}$ is the convolution. For a spherical test volume, the Percus-Yevick solution for hard spheres gives $\sigma^{2} /\langle N\rangle \cong S(0)+[1-S(0)]\langle\lambda N\rangle^{-1 / 3}$ for $\langle N\rangle>1 / \lambda$ and $\lambda \geq 0.1$ is a volume fraction dependent

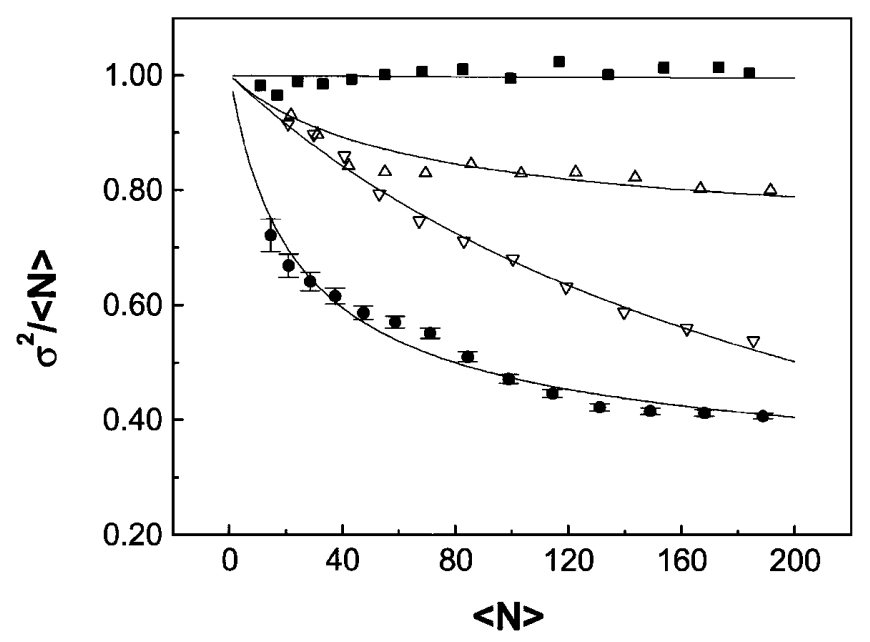

FIG. 3. Reduced number fluctuations, $\sigma^{2} /\langle N\rangle$, as a function of the average number of particles in the test volume, $\langle N\rangle$, shown for different times after mixing: 2 min (solid squares), $4 \mathrm{~h}$ (up triangles), $6 \mathrm{~h}$ (down triangles), and $8 \mathrm{~h}$ (solid circles). The solid curves from top to bottom represent Gaussian theory for $\omega=2$ and $\xi / a=200,33,68$, and 20 and $S(0)=0,0,-8$, and -0.53 , respectively. The error bars represent $68 \%$ confidence estimate of the average value. parameter. The $\langle N\rangle^{-1 / 3}$ dependence originates in the spherical test volume geometrical constraint and vanishes in the limit $S(0) \rightarrow 1$; it should not be confused with (and may indeed obscure) true $\langle N\rangle^{-1 / 3}$ number fluctuations.

Alternatively, we assume a simple model Gaussian pair correlation function:

$$
\begin{aligned}
g(\bar{r})= & 1-[1-S(0)] \\
& \times \exp \left[-\left(x^{2} \omega^{2 / 3}+y^{2} \omega^{2 / 3}+z^{2} \omega^{-4 / 3}\right) / \xi^{2}\right] \\
& \times v_{p} /\left(\pi^{3 / 2} \phi \xi^{3}\right),
\end{aligned}
$$

which gives $S(0)$ (nonthermodynamic) number fluctuations in the large test volume limit, represents a vertical to horizontal anisotropy proportional to $\omega$, can be evaluated easily in spherical (when isotropic) or rectangular coordinates, and like hard spheres has finite range $\xi$. For a rectangular test volume with dimensions $[X, Y, Z]$, the number fluctuations become

$$
\begin{aligned}
\frac{\sigma^{2}}{\langle N\rangle}= & 1-[1-S(0)] f\left(\omega^{1 / 3} X / \xi\right) f\left(\omega^{1 / 3} Y / \xi\right) \\
& \times f\left(\omega^{-2 / 3} Z / \xi\right) .
\end{aligned}
$$

The function $f(t)=\operatorname{erf}(t)-\left(1-e^{-t^{2}}\right) /(\sqrt{\pi} t)$ and ranges between zero as $t \rightarrow 0$ and unity as $t \rightarrow \infty$. If any one of the test volume dimensions tends to zero (or a small finite value), the number fluctuations tend to unity and are not suppressed (or not fully suppressed) by increasing the other test volume dimensions without limit. Figure 3 shows data compared to this functional form using experimental test volume dimensions and $\omega=2$. Fits at $2 \mathrm{~min}, 4 \mathrm{~h}$, and $8 \mathrm{~h}$ with $S(0)=0$ give $\xi / a=200,33$, and 13, respectively. Allowing $S(0)<0$ means number fluctuations become zero at finite $\langle N\rangle$ and give a fit to the data at $6 \mathrm{~h}$ with $S(0)=-7, \xi / a=68$ and at $8 \mathrm{~h}$ with $S(0)=-0.53, \xi / a=20$. Since $g(0)=0.92$ for $\xi / a=13$, increasing to 0.98 for $\xi / a=20$, and becoming essentially unity for larger values of $\xi / a$, the pair correlation may not prove measurably different from unity. Note that the theory with $S(0)=0$ approaches a limiting value $\sigma^{2} /\langle N\rangle \rightarrow 0.39$ (not zero) when increasing the average particle number by increasing viewing radius 


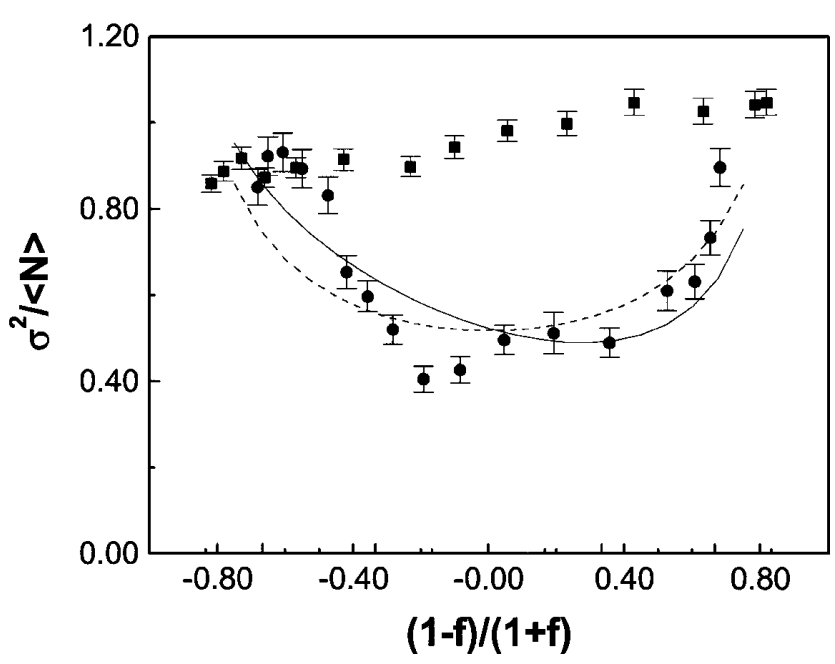

FIG. 4. Reduced number fluctuations, $\sigma^{2} /\langle N\rangle$, as a function of sample volume anisotropy, $(1-f) /(1+f)=(Z-X) /(Z+$ $X)$ with $\langle N\rangle=30$ at 2 min after mixing (open circles) and $8 \mathrm{~h}$ after mixing (closed circles). The dashed line represents $\xi / a=$ $13, \omega=1$ (isotropic) and the solid line $\omega=2$. The error bars represent $68 \%$ C.L. of the average value.

$\left(X=Z \cong \sqrt{\pi R^{2}}\right)$, because the light sheet thickness remains fixed and finite.

Finally Fig. 4 shows $\sigma^{2} /\langle N\rangle$ for a rectangular rather than circular test area with a horizontal to vertical length ratio, $f=X / Z$. Holding the product $X Z$ and the light sheet thickness $Y$ constant fixes the sample volume for a test of spatial anisotropy. If the number fluctuations are isotropic, the data will be symmetric when plotted against the variable $(1-f) /(1+f)$. The dashed curve gives the prediction for the isotropic $(\omega=1)$ Gaussian pair correlation function and the solid curve for $\omega=2$. The data are not symmetric, and the $\omega=2$ curve gives a slightly better fit than the isotropic curve.

In summary, number occupancy statistics, measured for non-Brownian particles settling in suspension, show significant suppression compared to purely random (Poisson) statistics. A previous study [20] failed to observe this suppression, because test volumes corresponding to $\langle N\rangle \sim 1$ were not sufficiently large. The initial random number fluctuations produce strong convection, which evidently reduces the number fluctuations in time. As these fluctuations reduce in magnitude, the velocity fluctuations diminish. The suppression develops over a long period of time and evidences anisotropy with a correlation length approximately $2 \times$ larger in the vertical direction. This should be compared with the dynamical anisotropy observed for velocity fluctuations $[7,13]$ and anticipated in simulations [21] and theoretically $[16,22]$. Comparison with a model correlation function suggests that the number fluctuations go to zero on sufficiently large length scales, thus preventing the divergence of velocity fluctuations with increasing sample size. The correlation range is many times the particle radius. But the effect on the pair correlation function (and structure factor) is small and similar in magnitude to the correlation range $\xi / a=10 \phi^{-1 / 3} \sim 63$ observed for velocity fluctuation [7], while the number fluctuations decrease monotonically in time, the Gaussian model amplitude and correlation range values fluctuate less systematically. Future work will explore the approach to steady state or asymptotic limit.

We thank NASA for supporting this work through Grant No. NAG3-1852.

[1] W. B. Russel, D. A. Saville, and W. R. Schowalter, Colloidal Dispersions (Cambridge University Press, Cambridge, 1989), Chap. 12.

[2] J. M. Burgers, Proc. K. Ned. Akad. Wet. 44, 1045 (1942).

[3] G. K. Batchelor, J. Fluid Mech. 52, 245 (1972).

[4] E. J. Hinch, J. Fluid Mech. 83, 695 (1977).

[5] M. P. Brenner, Phys. Fluids 11, 754 (1999).

[6] R.E. Caflisch and J.H.C. Luke, Phys. Fluids 28, 259 (1985).

[7] P. N. Segrè, E. Herbolzheimer, and P. M. Chaikin, Phys. Rev. Lett. 79, 2574 (1997).

[8] E. J. Hinch, in Disorder and Mixing, edited by E. Guyon et al. (Kluwer Academic, Dordrecht, 1988), p. 153.

[9] A. J. C. Ladd, Phys. Rev. Lett. 76, 1392 (1996).

[10] A. J. C. Ladd, Phys. Fluids 9, 491 (1997).

[11] J. M. Ham and G. M. Homsy, Int. J. Multiphase Flow 14, 533 (1988).

[12] H. Nicolai and E. Guazzelli, Phys. Fluids 7, 3 (1995).

[13] H. Nicolai, B. Herzhaft, E. J. Hinch, L. Oger, and E. Guazzeli, Phys. Fluids 7, 12 (1995).

[14] D. L. Koch and E. S. G. Shaqfeh, J. Fluid Mech. 224, 275 (1991).

[15] P. Tong and B. J. Ackerson, Phys. Rev. E 58, 6931 (1998).

[16] A. Levine, S. Ramaswamy, E. Frey, and R. Bruinsma, Phys. Rev. Lett. 81, 5944 (1998).

[17] J. P. Hansen and I. R. McDonald, Theory of Simple Liquids (Academic, London, 1986).

[18] M. Wertheim, Phys. Rev. Lett. 8, 321 (1963); E. Thiele, J. Chem. Phys. 38, 1959 (1963).

[19] B. J. Berne, Dynamics of Charged Macromolecules in Solution, in Photon Correlation Spectroscopy and Velocimetry (Plenum Press, New York, 1976); P. N. Pusey, J. Phys. A 8, 1433 (1975).

[20] T. N. Smith, J. Fluid Mech. 32, 203 (1968).

[21] A. J. C. Ladd, Phys. Fluids 5, 299 (1993).

[22] D. L. Koch, Phys. Fluids 5, 1141 (1993). 